\title{
High-power microwave diplexers for advanced ECRH systems
}

\author{
W. Kasparek ${ }^{1}$, M. Petelin ${ }^{2}$, V. Erckmann ${ }^{3}$, A. Bruschi ${ }^{4}$, F. Noke ${ }^{3}$, F. Purps ${ }^{3}$, F. Hollmann ${ }^{3}$, Y. \\ Koshurinov $^{2}$, L. Lubyako ${ }^{2}$, B. Plaum ${ }^{1}$, W. Wubie ${ }^{1}$, ECRH groups at IPP Greifswald ${ }^{3}$, FZK \\ Karlsruhe $^{5}$, IFP Milano ${ }^{4}$, IAP Nizhny Novgorod ${ }^{2}$, and IPF Stuttgart ${ }^{1}$ \\ 1. Institut für Plasmaforschung, Universität Stuttgart, Pfaffenwaldring 31, D-70569 Stuttgart, Germany \\ 2. Institute of Applied Physics, RAS, 603950 Nizhny Novgorod, Russia \\ 3. Max-Planck-Institut für Plasmaphysik (IPP), EURATOM-Association, D-17491 Greifswald, Germany \\ 4. Istituto di Fisica del Plasma, EURATOM-ENEA-CNR Ass., via R. Cozzi 53, 20125 Milano, Italy \\ 5. Forschungszentrum Karlsruhe, Ass. EURATOM-FZK, IHM, D-76021 Karlsruhe, Germany
}

\begin{abstract}
In Electron cyclotron resonance heating systems, high-power multiplexers can be employed as power combiners, adjustable power dividers, fast switches to toggle the power between two launchers, as well as frequency sensitive directional couplers to combine heating and diagnostic applications on one launcher.

In the paper, various diplexer designs for quasi-optical and corrugated waveguide transmission systems are discussed. Numerical calculations, low- power tests and especially high-power experiments performed at the ECRH system of W7-X are shown, which demonstrate the capability of these devices. Near term plans for applications on ASDEX Upgrade and FTU are presented. Based on the present results, options for implementation of power combiners and fast switches in the ECRH system of ITER is discussed.
\end{abstract}

Key words: Diplexer, Power combiner, Millimeter Wave transmission, Electron cyclotron Heating.

PACS numbers: 52.50.Gj, 52.35.-g, 84.40.-x, 42.79.Ci 


\section{Introduction}

Electron cyclotron resonance heating is of high importance as an effective and flexible heating system for tokamaks and stellarators. Technology for sources, transmission and launchers is highly developed. Further improvements can be obtained by introduction of high-power diplexers $[1,2,3,4,5,6]$ which might be used for several applications, leading to an increase of flexibility, performance or to a simplification of the system.

Combining of powers from two (or more) conventional gyrotrons would essentially reduce the number of transmission lines and launchers for ECRH systems of large fusion devices, e.g. ITER. High-power combiners (multiplexers) can be composed of oversized four-port diplexers, each representing a kind of interferometer or quasi-optical resonator with corrugated mirrors; the gyrotrons being implied to generate slightly different frequencies.

In any gyrotron, modulation of a voltage results in a small, within some tens of $\mathrm{MHz}$, shifting of the radiation frequency. This shift can provide, by using an adequate multiplexer, switching of the combined wave beam between output channels. In particular, fast, non-mechanical, electronically controlled switching of the power from continuously operating gyrotrons between two antennas synchronous to the rotation of the magnetic islands in the tokamak plasma would maximize the efficiency for stabilization of neo-classical tearing modes [7]. Additional scenarios for multi-beam combining and controlled switching of the combined beam could be offered by using phase-controlled gyrotrons (if the latter ones were developed at frequencies and powers of interest).

The reciprocity and filter characteristics of diplexers are favourable for sharing the same antenna to combine high-power ECRH launching with reception of a low-power plasma diagnostic signal (e.g. ECE) [8].

In the paper, various diplexer designs for quasi-optical and corrugated waveguide transmission systems including numerical and experimental results are presented. Results from fast switching of a high-power beam between two output channels using a gyrotron, and recent experiments for power combination of two gyrotrons from the ECRH system for the stellarator W7-X are reported. The application of diplexers for synchronous MHD-mode stabilization at ASDEX Upgrade and FTU are discussed. Finally, the possible implementation of power combiners and fast switches in the ECRH system of ITER is discussed.

\section{Designs for high-power diplexers}

Frequency diplexers can be designed in various forms. Basically, these diplexer are four-port devices consisting of a directional coupler (hybrid) at the input, a delay-line used in single-path or in multi-path), and a second hybrid at the output. Well-known designs compatible with highpower millimetre waves are two-beam interferometer (e.g. the Mach-Zehnder type [2,6]), multibeam interferometers (Fabry-Perot type, or ring resonator $[1,3]$ ) and combinations thereof. As high-power compatible hybrids, low-loss dielectric beam splitters [2,6], metallic phase gratings (magic Y) [4,10], and square corrugated waveguides [5,11] have been identified up to now. In principle, any of the solutions found can be integrated into corrugated waveguide as well as in beam waveguide transmission systems by using appropriate interface elements. In Fig. 1, we show three possible designs with the corresponding transmission characteristics, being periodic with $\Delta f_{\mathrm{F}}=c / L$. Details can be found in the references given above. 

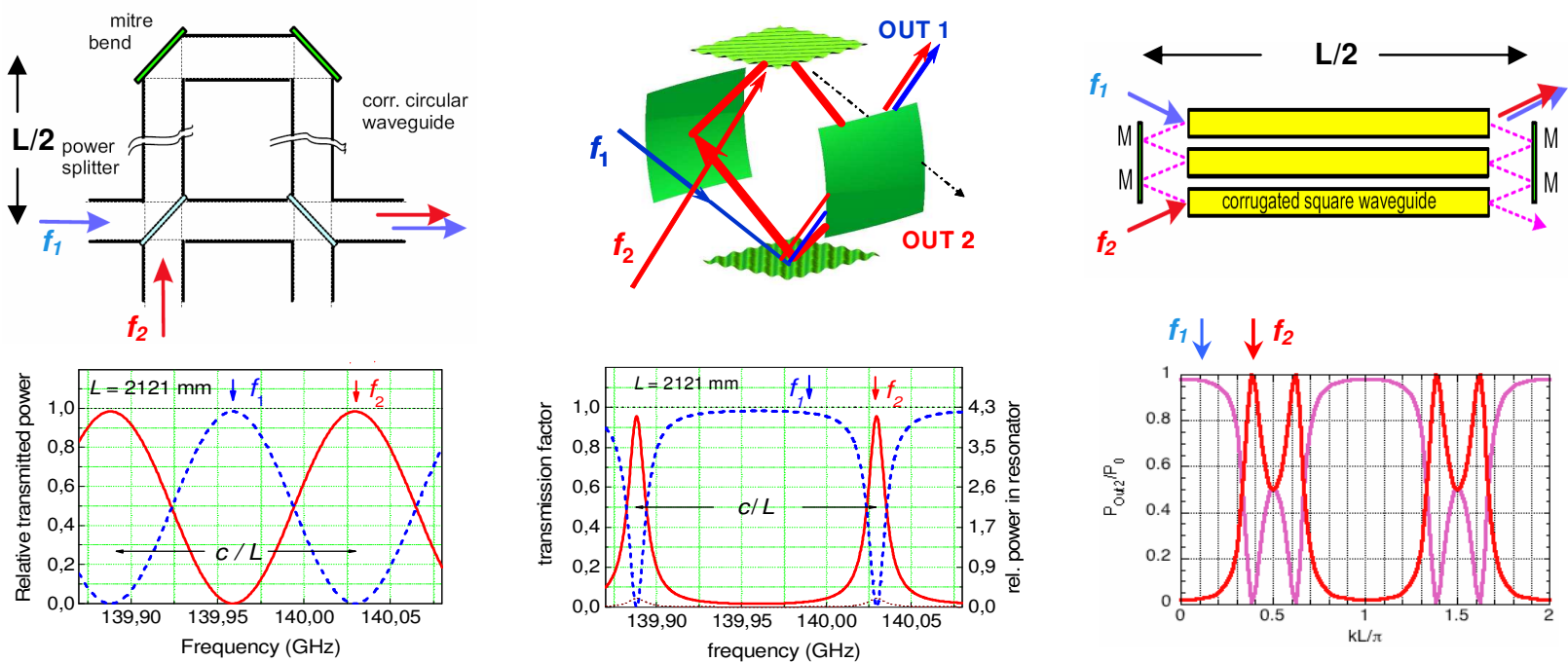

Fig. 1:

Possible designs of high-power diplexers and the corresponding transmission functions. a) MachZehnder type interferometer with dielectric beam splitters; $b$ ) ring resonator (length $L$ ) with coupling gratings; c) two-loop diplexer using square waveguide splitters. Arrows illustrate the situation for power combination.

The Mach-Zehnder type diplexer shown in Fig. 1a [6] consists of two dielectric power splitters integrated into $\mathrm{HE}_{11}$ waveguide with a direct connection and a delay line with a length difference $\mathrm{L}$ with respect to the direct line. For the angle of incidence of $45^{\circ}$ and s-polarization, CVD diamond is an appropriate material for a high-power 3-dB splitter. For strongly oversized systems, e.g. $170 \mathrm{GHz}$ and $\varnothing 63.5 \mathrm{~mm}$ waveguide, the theoretical transmission loss is very low. By choosing the appropriate L, the sinusoidal transmission characteristics can be easily matched to the application envisaged.

The resonant diplexer in Fig $1 \mathrm{~b}$ is a 4-mirror ring resonator $[2,3,9]$ consisting of two focussing mirrors and two plane phase gratings for coupling of the incident beams to the resonator via the $-1^{\text {st }}$ diffraction order. Additional mirrors provide matching to the input and output beam or waveguide. The transmission characteristics are defined by the grating efficiency and the path length $L$ of one round-trip in the resonator. Special features are the relatively narrow resonant transmission channels separated by broad non-resonant bands. Transmission loss of a few percent [3] affects mainly the resonant channel due to absorption of the mirrors in the resonator.

The two-loop resonant diplexer (Fig. 1c) consists of two nested loops [5] formed by square corrugated waveguides with length $2 a^{2} / \lambda$ used as 3 -dB hybrids [11], and the reflectors $\mathrm{M}$. Additional mirrors provide matching of the inputs and outputs to the transmission system. The periodicity of the transmission function is determined by the overall length $\mathrm{L} / 2$ of the system. Special features are the steep slopes and the double-humped structure of the resonant channels. Some insertion loss occurs in the waveguides and due to the coupling between the guides via the mirrors [12]. 


\section{Experimental investigations}

For the high-power diplexer concepts discussed before and others [2], test systems have been built and investigated with low power. Within the experimental possibilities of these mock-ups, a good agreement with theory is obtained for all set ups. Details can be found in the respective references [2-12].

The quasi-optical resonant diplexer was as well exposed to high-power tests, which were performed at the ECRH system for the stellarator W7-X [13]. For this purpose, the diplexer was integrated into the beam duct with the help of matching optics, such that one or two gyrotrons (B1 and B5) could be fed into the inputs, and the outputs were connected to cw calorimetric loads. Experiments were performed with power around $500 \mathrm{~kW}$ and pulse lengths limited to several seconds by the un-cooled mirrors in the mock-up diplexer.

By modulating the body voltage $\mathrm{U}_{\mathrm{B}}$ (square wave, $\Delta \mathrm{U}_{\mathrm{B}} \leq 5 \mathrm{kV}, f_{\text {mod }} \leq 20 \mathrm{kHz}$ ), frequency-shift keying of the gyrotron $\mathrm{B} 1$ with $\Delta f_{\mathrm{gyr}} \leq 30 \mathrm{MHz}$ could be obtained. This allowed to toggle the power in the rhythm of the frequency shift between the two outputs with a contrast of better than $90 \%$, as shown in Fig. 3 for the example for $f_{\bmod }=5 \mathrm{kHz}[3]$.

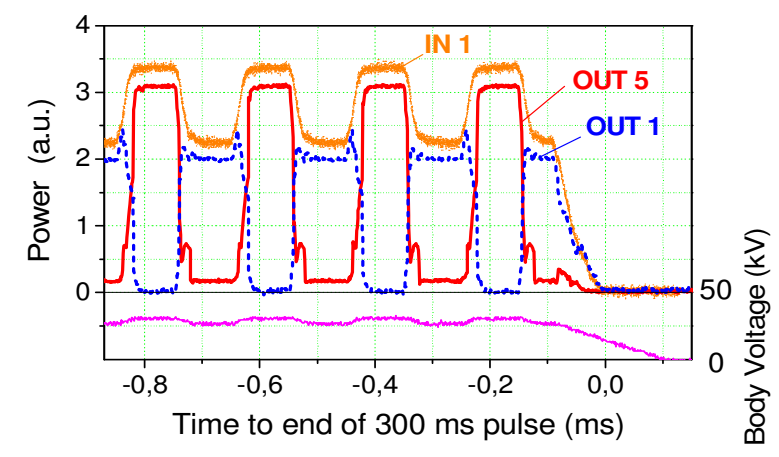

Fig. 2:

Power signals from output 1 (blue, dashed) and output 2 (red, solid) from the diplexer, shown at the end of a 300 ms pulse with $U_{a c c}=81.5 \mathrm{kV}, \Delta U_{B}=4 \mathrm{kV}, f_{M O D}=5 \mathrm{kHz}$ square wave. The enveloping trace (orange) is the signal from the gyrotron power monitor of gyrotron B1. The lower trace shows the body voltage.

Power combination was demonstrated by feeding two gyrotrons into the diplexer [14]. It was tuned such that a resonance was coincident with the frequency of gyrotron B1 (after thermalization of the cavity). The frequency of gyrotron B5 was set about $40 \mathrm{MHz}$ below the frequency of B1 using the dependence of the cavity temperature and thus the frequency from the generated power. A $10 \mathrm{~s}$ pulse (Fig. 4) showed a power combination with an efficiency of about $90 \%$. By modulating the frequency of the two gyrotrons, toggling of the combined power between the outputs in the $\mathrm{kHz}$ range could be demonstrated [14]. 


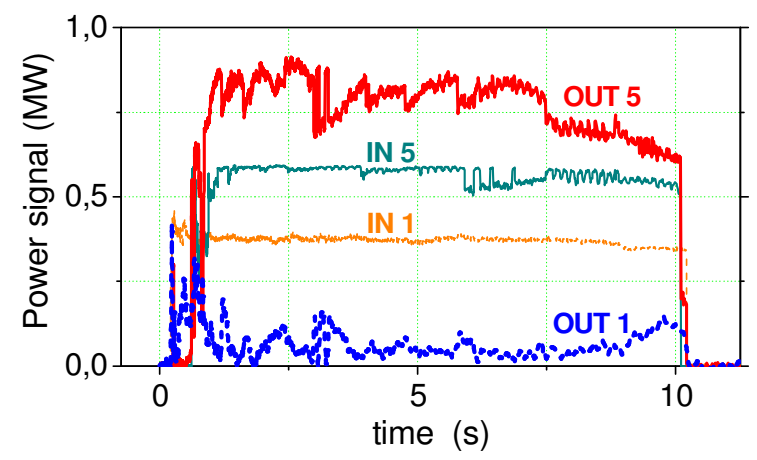

Fig. 3:

Power signals from a 10-s power combination experiment. IN1 and IN5: Power of gyrotrons B1 and B5, OUT 1 and OUT 5: output power from the diplexer coupled to the loads. Residual power in OUT 1 is mainly caused by spontaneous frequency jumps of the BI gyrotron and an increase of the B1 frequency during the end of the pulse [13]. Note that the signals suffer somewhat from interference with stray radiation, and therefore are calibrated by comparing the averages over the pulse with corresponding calorimetric measurements.

\section{Applications of high-power diplexers}

\subsection{Frequency tracking}

For the operation of any diplexer in an ECRH system, a remotely controllable, preferably automatic tuning of its frequency characteristics over one period of the transmission curve, i.e. $\Delta f_{\mathrm{F}}=c / L$, is needed to match the gyrotron frequency and to compensate for thermal drifts. This means that one mirror in the resonator or delay line is equipped with a drive to move the mirror in normal direction over a distance of less than a wavelength. Fast and precise drive concepts for this purpose are in development [8]. Note that the misalignment due to this frequency tracking is negligible for all diplexer designs discussed here.

\subsection{Near-term plans for applications in ASDEX upgrade and FTU.}

At the new ECRH system for ASDEX Upgrade [15], the implementation of a resonant diplexer prototype is in preparation. This diplexer (Fig.4) features a compact design within a rigid box providing stable alignment as well as microwave shielding. Corrugated waveguide inputs and outputs are matched to the resonator mode by $\mathrm{HE}_{11}-\mathrm{TEM}_{00}$ mode transitions, and polarizers in the mitre bends at the output waveguides are inserted to match the polarization to the launching conditions. A mirror drive allows the tuning of the resonator. The main application to be tested is AC-stabilization of NTMs by toggling of the power between two launchers, which aim at poloidal positions being displaced by $180^{\circ}$ with respect to the phase of the NTMs; thus the driven EC current is always induced in the O-points yielding maximum NTM suppression [7]. A further application will be an in-line ECE system, where ECRH and ECE will be applied sharing the same launcher and thus a common line of sight [8]. 


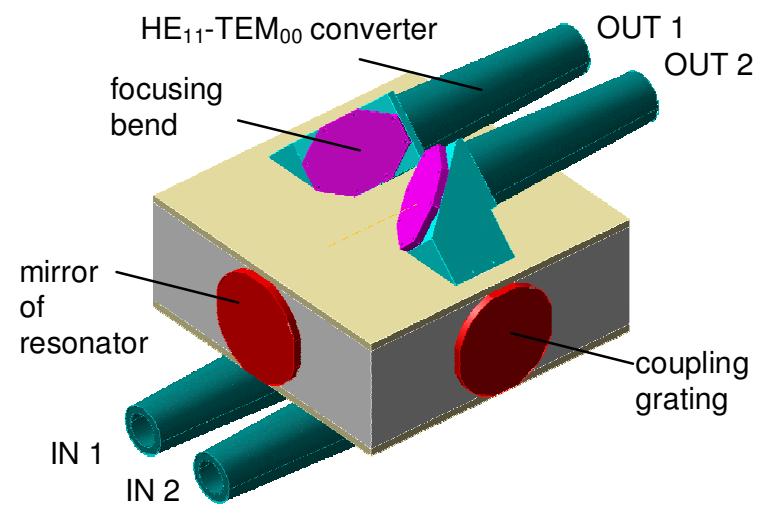

Fig. 4:

Conceptual design of a compact resonant diplexer with corrugated waveguide ports, as designed for tests in the new ECRH system at ASDEX Upgrade.

At FTU, the frequency-to-voltage characteristics of the GYCOM gyrotrons are favourable [2]. A diplexer/combiner is proposed as switcher between two poloidally symmetric antennas, in order to perform high-efficiency tearing mode stabilization experiments. Combining pairs of beams in a single line would also allow for fitting the 4 available sources into the new envisaged FTU ECRH launcher $[16,17]$ equipped with 2 antennas. The resonator version of Fig 1.c with squared corrugated waveguides [5] is designed for insertion in the long run of the 4 parallel transmission lines. Input matching optics converts beams to a smaller size before entering the combiner. Highpower tests of a prototype, now in final design stage (Fig. 5), are planned first.

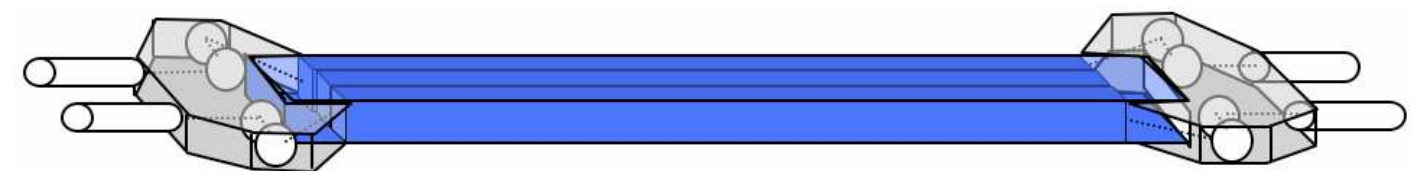

Fig. 5:

Sketch of the resonant diplexer/combiner made with 3 parallel squared corrugated waveguides (in colour) and input/output optics (in grey) proposed for tests in the ECRH system at Frascati Tokamak Upgrade.

\subsection{Options for applications and integration in the ECRH system for ITER}

The basic ITER ECRH system [18] will employ 24 170-GHz gyrotrons. It is assumed, that these will be free-running oscillators (although gyro-amplifiers and phase-locking schemes are under development, but at present do not provide sufficient power). The gyrotrons are connected via evacuated corrugated $\mathrm{HE}_{11}$ waveguide to 3 upper launchers [19] (UL; 8 beams each, mainly for NTM stabilization) and one equatorial launcher (EL; 24 beams, mainly for central heating and current drive). A central unit with mechanical waveguide switches allows connecting the gyrotrons either to the ULs or the EL.

As a basic modification, the mechanical waveguide switches can be replaced by diplexers. Firstly, this allows switching of the power from the EL to the UL by re-tuning of the diplexer as 
before, but without the need to switch off the gyrotrons during the switching process. Note that arbitrary distribution of the power to the launchers is possible, as the diplexer has no undefined position like a conventional switch. Secondly, efficient AC-stabilization of NTMs is possible as soon as a mode occurs: The gun anode or body voltage of the gyrotrons is modulated (by a few $\mathrm{kV}$ only) with the mode frequency, and the diplexers are tracked such that the corresponding frequency modulation results in a maximum power modulation at the outputs for the ULs, synchronous to the rotation of the island. The asynchronous power is still available at the EL and can be used to continue the task as before the onset of the NTMs. Thirdly, if (at a later stage) more gyrotrons are to be added to the system, they could be fed into the second inputs of the diplexer to provide power combination. Here one has to choose pairs of gyrotrons with a frequency difference (for optimal output in steady state operation) not equal to (a multiple of) the free spectral range of $\Delta f_{\mathrm{F}}=c / L$, the ideal case being a difference of (an odd multiple of) roughly

$\Delta f_{\mathrm{F}} / 2$ ( $c f$. Fig. 1). The diplexer is tuned such that a resonance frequency $f_{2}$ coincides with $f_{\mathrm{A}, 0}$, the optimal frequency of gyrotron $\mathrm{A}$; thus, the optimal frequency of gyrotron $\mathrm{B}, f_{\mathrm{B}, 0}$, stays always non-resonant. To direct the combined power to the other output channel, the tracking of the diplexer resonance has to be switched to $f_{\mathrm{B}, 0}$. In case of operation as a fast switch and combiner, both gyrotrons are frequency modulated with sufficient $\Delta f_{\mathrm{S}}$, and the diplexer is tracked that $f_{\mathrm{A}, \mathrm{O}}$ (or $f_{\mathrm{B}, \mathrm{O}}$ ) is coincident with $f_{2}$.

Further options are the replacement of switches between launchers and loads, allowing gyrotrons in hot stand-by, and diplexers placed near to the generator hall for power combination from two 1-MW gyrotrons on a common transmission line.

Several notes need to be added to these applications. Firstly, although possibly all types of diplexers can be designed for arbitrary polarization, the different launching conditions for the EL and the UL will most probably require different polarizations. Therefore, for fast switching applications, the diplexer should be installed between the gyrotrons and the polarizers. Secondly, for about $0.5 \mathrm{~s}$ after switch-on of the gyrotrons, where the frequency varies rapidly due to thermal cavity expansion, the routing of the power probably cannot be controlled. This is, however, no problem for hot stand-by operation. Thirdly, the diplexers discussed here differ strongly with respect to the transmission characteristics, insertion loss, cross-talk, dimensions and requirements for system integration. Therefore, the diplexer concept chosen will depend on the concrete tasks to be performed, and on the experimental environment. A detailed discussion is beyond the scope of this paper.

\section{Conclusions}

It has been shown, that high-power diplexers are a valuable component, which can strongly increase the performance and flexibility of ECRH systems. The results obtained up to now in the development of several prototypes including the high-power demonstration of fast switching power combination from two gyrotrons confirm the applicability of these devices. In conclusion, the results motivate the development of power combiners and fast switches until maturity, especially in view to applications in ITER and other next step devices.

Further research is aimed to study other multiplexing schemes and arrays of phase-controlled gyrotrons. Gyrotron developers are encouraged to continue the development of frequency or even phase controlled gyrotrons, the availability of which would further extend the application palette of high-power diplexers. 
This work is carried out in the frame of the virtual institute "Advanced ECRH for ITER" (collaboration between IPP Garching and Greifswald, FZK Karlsruhe, IHE Karlsruhe, IPF Stuttgart, IAP Nizhny Novgorod, and IFP Milano), which is supported by the HelmholtzGemeinschaft deutscher Forschungszentren.

\section{References}

1. M.I. Petelin and W. Kasparek, Electrically controlled scanning of wave beam produced by gyrotron: Option for plasma fusion experiment. Proc. 6th Int. Vacuum Electronics Conference (IVEC 2005), Noordwijk, The Netherlands, p. 131.

2. W. Kasparek et al., Fast switching and power combination of high-power electron cyclotron wave beams: principles, numerical results and experiments. Fusion Sci. Technol. 52 (2007) 281 - 290, and references therein.

3. W. Kasparek et al., A fast switch, combiner and narrow-band filter for high-power millimetre wave beams. Nucl. Fusion 48 (2008) 054010.

4. F. M. A. Smits, Power combiners for incoherent waves. Proc. of 8th Joint workshop on ECE and ECRH, Report IPP III/186, Vol. 2, 607 - 621, 1993.

5. A. Bruschi et al., Beam Combination and Routing at High Power with a Ring-type Waveguide Millimeter-Wave Resonator, Fusion Sci. Technol. 53 (2008), 97 - 103.

6. W. Wubie et al., Numerical and experimental investigations of a diplexer for combination and switching of high-power millimeter waves, Int. Conf. on Infrared and Millimeter Waves, Pasadena, 2008.

7. M. Maraschek, et al., Enhancement of the Stabilization Efficiency of a Neoclassical Magnetic Island by Modulated Electron Cyclotron Current Drive in the ASDEX Upgrade Tokamak Phys. Rev. Lett. 98, 025005 (2007).

8. W. Bongers et al, Feedback Stabilization of Tearing Modes by Electron Cyclotron Current Drive controlled by in-line Electron Cyclotron Emission, submitted to Fusion Sci. Technol. (2008).

9. Yu. I. Koshurinov et al., Diplexer based on open cavity with corrugated mirrors. Tech. Phys. Lett. 31 (2005) $709-711$.

10. Petelin M I, Quasi-optics in High-Power Millimeter-Wave Systems. 6th Workshop on High Energy Density and High Power RF, WV, USA, AIP Conference Proc. 691 (2003), 251-262.

11. S.V. Kuzikov, "Wave beam multiplication phenomena to RF power distribution systems of highenergy linear accelerators". Int. J. Infrared Millimeter Waves 19 (1998), 1523-1539.

12. O. D'Arcangelo, et al., Tests of a $105 \mathrm{GHz}$ prototype diplexer combiner based on square corrugated waveguide, this conference.

13. V. Erckmann et al., 2007, Electron Cyclotron Heating for W7-X: Physics and Technology. Fusion Sci. Technol., 52 (2007) 291.

14. V. Erckmann, et al, Power Combination of Two $140 \mathrm{GHz}$ Gyrotrons and Fast Switching of the Combined Beam, accepted for publication, Fusion Sci. Technol. (2008). 
15. D. Wagner et al., The new multifrequency electron cyclotron resonance heating system for ASDEX Upgrade. Fusion Sci. Technol. 52 (2007), 313 - 320.

16. A. Bruschi et al., "A new Fast-steerable ECRH Launcher for FTU”, submitted to Fusion Sci. Technol., (2008).

17. W. Bin et al. "Design of a new ECRH launcher for FTU tokamak", this Conference.

18. Design Description Document for the ITER ECRH System G 52 DDD 5 01-05-29 W 0.1.

19. R. Heidinger et al., Conceptual design of the ECH upper launcher system for ITER, this conference. 